\title{
Regulation of MHC class II-peptide complex expression by ubiquitination
}

\section{Kyung-Jin Cho and Paul A. Roche*}

Experimental Immunology Branch, National Cancer Institute, National Institutes of Health, Bethesda, MD, USA

\section{Edited by:}

Laura Santambrogio, Albert Einstein

College of Medicine, USA

\section{Reviewed by:}

Ulrike Seifert,

Charité-Universitätsmedizin Berlin, Germany

Hans Acha-Orbea, Center of Immunity and Infection Lausanne, Switzerland

\section{*Correspondence:}

Paul A. Roche, Experimental

Immunology Branch, National Cancer Institute, National Institutes of Health, Building 10, Room 4B36, Bethesda, MD 20892, USA

e-mail:paul.roche@nih.gov
MHC class II (MHC-II) molecules are present on antigen presenting cells (APCs) and these molecules function by binding antigenic peptides and presenting these peptides to antigenspecific $\mathrm{CD} 4^{+} \mathrm{T}$ cells. APCs continuously generate and degrade MHC-II molecules, and ubiquitination of MHC-II has recently been shown to be a key regulator of MHC-II expression in dendritic cells (DCs). In this mini-review we will examine the mechanism by which the E3 ubiquitin ligase March-I regulates MHC-II expression on APCs and will discuss the functional consequences of altering $\mathrm{MHC}$-II ubiquitination.

\section{Keywords: MHC class II, March-I, ubiquitination, degradation, endocytosis, recycling}

Major histocompatibility complex class II molecules (MHC-II) function by presenting processed antigens, derived primarily from exogenous sources, to $\mathrm{CD} 4^{+}$T-lymphocytes. MHC-II molecules thereby are critical for the initiation of the antigen-specific immune response. MHC-II is constitutively expressed by immune cells including $\mathrm{B}$ cells, monocytes, macrophages, and dendritic cells (DCs) and even non-hematopoietic cells can express MHC-II under inflammatory conditions. While each of these MHC-IIbearing cell types function as "professional" antigen presenting cells (APCs), DCs have received much attention as APCs since it is these APCs that are able to stimulate naïve antigen-specific $\mathrm{CD} 4^{+} \mathrm{T}$ cells. Tissue-resident DCs have often been referred to as the "sentinels of the immune system," and it is their job to continuously sample their microenvironment by internalizing extracellular fluid and generating peptide-MHC-II complexes (pMHC-II) that can potentially interact with antigen-specific T cells (1). While resting (immature) DCs do express considerable amounts of pMHC-II on their surface, stimulation of DCs by a variety of inflammatory stimuli results in increased expression of pMHC-II at the plasma membrane by at least two mechanisms: (1) by increasing antigen proteolysis/peptide binding to MHC-II $(2,3)$ and (2) by promoting pMHC-II movement from intracellular antigen processing compartments to the cell surface $(4,5)$. Activation of DCs transiently increases MHC-II synthesis and increases macropinocytosis. However, within hours of the activation signal CIITA synthesis (and thus MHC-II synthesis) is severely reduced and macropinocytosis is terminated (6, 7). Together, these processes poise the recently activated DC to generate large amounts of pMHC-II with antigens derived from pathogens at the site of infection, thereby enhancing their ability to stimulate antigen-specific $\mathrm{CD} 4^{+} \mathrm{T}$ cells.

At steady-state, the rate of generation of pMHC-II complexes in immature DCs is equal to the rate of pMHC-II degradation.
It is regulation of pMHC-II degradation that is the topic of this mini-review. Recently, it has been shown that ubiquitination participates in pMHC-II degradation (8,9). MHC-II is ubiquitinated on a single conserved lysine in the cytoplasmic domain of the MHC-II $\beta$-chain present in mouse I-A and I-E molecules as well as human HLA-DR molecules, heretofore referred to as K225. The membrane-associated RING-CH-domain containing E3 ubiquitin ligase March-I is the sole E3 ligase responsible for the ubiquitination MHC-II in B cells and is the primary E3 ligase responsible for ubiquitination of MHC-II in DCs (10). March-I expression is highly enriched in secondary lymphoid tissues such as spleen and lymph node (11) and appears to be especially prominent in APCs such as B cells (10), DCs (12-14), and monocytes (15).

Expression of March-I leads to the down-regulation of several surface molecules including MHC-II, CD86, and transferrin receptor (TfR) $(11,16)$. In March-I-deficient B cells, MHC-II expression is much higher than in control B cells, and this effect was mediated by ubiquitination of K225 in the I-A $\beta$-chain (10). Gainof-function experiments in which March-I was overexpressed in MHC-II-expressing HeLa-CIITA cells or human monocytederived DCs (MoDC) resulted in profound down-regulation of surface HLA-DR level in these cells $(14,16)$. Our own loss-offunction experiments revealed that expression of MHC-II was significantly higher on immature DCs isolated from March-I KO mice than on DCs isolated from WT mice. Essentially identical results were obtained using DCs isolated from MHC-II $\mathrm{K}_{255} \mathrm{R}$ ubiquitination-mutant mice, demonstrating that ubiquitination of MHC-II K225 by March-I regulates MHC-II surface expression (14).

Whereas March-I is constitutively expressed in resting professional APCs, March-I can be induced or repressed by different stimuli both in vitro and in vivo. Infection of mouse macrophages with Francisella tularensis induces the ubiquitin-dependent 
degradation of MHC-II by promoting IL-10-dependent March-I expression (17). IL-10 up-regulate March-I expression and MHCII ubiquitination not only in mouse macrophages but also in human monocytes and mouse B cells $(18,19)$. Curiously, although most MHC-II-expressing APCs constitutively express March-I, interferon-gamma-treatment of monocytes, which leads to MHCII expression, does not result in March-I expression unless the cells are also treated with IL-10, highlighting the complexity of March-I expression in APCs. Curiously, the ability of IL-10 to downregulate MHC-II expression in DCs is due to induction of March-I $(15,20)$. Perhaps more important than the up-regulation of basal MarchI expression, March-I mRNA expression is significantly reduced when resting APCs are stimulated with toll-like receptor (TLR) signals such as LPS, PGN, poly (I:C) $(16,21)$. The March-I protein has a half-life of less than $30 \mathrm{~min}$, potentially regulated by autoubiquitination (12), therefore the termination of March-I mRNA expression leads to a rapid drop in March-I protein levels (16). The reduction of March-I protein expression upon DC activation has profound consequences for MHC-II ubiquitination, for upon DC activation MHC-II ubiquitination is dramatically reduced (8-11, 21). As will be discussed below, it is the activation-induced termination of March-I expression that primarily regulates MHC-II surface expression in DCs.

The available evidence shows that ubiquitination by March-I is an important regulator of MHC-II degradation. Simple overexpression of March-I dramatically reduces the survival of MHC-II molecules in HeLa-CIITA cells and in B cells $(10,14,16)$. In addition, studies in mutant mice have shown that surface MHC-II expression is higher and the half-life of MHC-II is significantly prolonged in B cells isolated from March-I KO mice as compared to WT mice (10). A similar role for human March-I in regulation of HLA-DR expression MoDCs has also been described (16). We have shown that surface pMHC-II complexes on March-I KO DCs or $\mathrm{K}_{255} \mathrm{R}$ ubiquitination-mutant immature DCs are considerably more stable than those in WT DCs and kinetic analyses demonstrated that ubiquitination directly affects the rate of degradation of surface pMHC-II (14). Limiting lysosomal proteolysis delays March-I-induced MHC-II degradation in DCs (9), suggesting that ubiquitinated MHC-II is degraded in late endosomes/lysosomes in these cell types.

In immature DCs, a relatively large pool of MHC-II is present in intracellular antigen processing compartments. During TLRmediated DC activation many of these MHC-II molecules traffic to and accumulate on the plasma membrane (3). Maturation of DCs not only inhibits fluid-phase macropinocytosis in DCs (22, 23), but also inhibits the kinetics of MHC-II endocytosis from the cell surface in human MoDCs (6). The findings that MHC-II is (1) ubiquitinated in immature DCs, (2) internalizes efficiently in immature DCs, and (3) accumulates intracellularly in immature DCs (but not mature DCs) has led to speculation that ubiquitination regulates MHC-II endocytosis in DCs. It has been shown that anti-MHC-II mAb accumulate intracellularly in WT immature DCs but not in $\mathrm{K}_{255} \mathrm{R}$ ubiquitination-mutant immature DCs $(8,9,14)$, a finding that is consistent with the hypothesis that ubiquitination regulates MHC-II endocytosis.

However, the role of ubiquitination in enhancing the kinetics of MHC-II internalization remains controversial. De Gassart et al. have reported that MHC-II internalization was reduced by $50 \%$ in MoDCs in which March-I expression was reduced by transfected siRNA (16). By contrast, our own studies in both human and mouse DCs have shown that while MHC-II endocytosis is slightly more rapid in immature DCs than in mature DCs, there is no difference in the kinetics of MHC-II endocytosis in DCs from WT, March-I KO, and MHC-II $\mathrm{K}_{255} \mathrm{R}$ ubiquitination-mutant mice (14). Furthermore, analysis of March-I-deficient B cells revealed that the internalization rate of MHC-II in March-I KO B cells was similar to that in WT B cells, demonstrating that MHC-II ubiquitination is not required for internalization of MHC-II in B cells (10). We have also examined the kinetics of endocytosis of MHCII in HeLa-CIITA cells expressing (or not) March-I. In agreement with our results in DCs, we found no difference in the rate of MHC-II endocytosis in HeLa-CIIA cells expressing GFP alone or GFP-March-I, demonstrating that ubiquitination of MHC-II does not affect the kinetics of MHC-II endocytosis in DCs (14). These data showing that ubiquitination does not affect MHC-II endocytosis rate are also consistent with similar types of experiments showing that ubiquitination profoundly affects the intracellular distribution of fibroblast growth factor receptor 1 and epidermal growth factor receptor but does so without affecting the kinetics of receptor endocytosis $(24,25)$.

Despite the significant effects of March-I on pMHC-II ubiquitination and pMHC-II localization, we do not yet have a clear understanding of how ubiquitination actually regulates the stability of pMHC-II complexes. Recently it has been found that the MHC-II polyubiquitin chain length is different in DCs and in B cells and that longer polyubiquitin chains (such as those present in DCs) promote more efficient MHC-II lysosomal targeting (26). How polyubiquitin chain length is regulated in APCs (whether by diminished ubiquitination or enhanced activity of deubiquitinating enzymes) remains to be determined. Clearly ubiquitination of MHC-II regulates MHC-II surface expression, and while our own data argues that ubiquitination does not directly affect pMHC-II endocytosis rate, the possibility exists that ubiquitination affects $\mathrm{MHC}-\mathrm{II}$ surface expression by regulating the ability of pMHC-II to recycle back to the plasma membrane after endocytosis. pMHC-II complexes continuously internalize and recycle from early endosomes to the plasma membrane and back again (27). While analysis of internalization rate data has suggested that pMHC-II recycling rates are different in immature DCs and mature DCs (6), we have been unable to find direct experimental data to support this theory. We have recently shown that internalized pMHC-II enters into elongated Arf6 ${ }^{+} \mathrm{Rab} 5^{+}$tubular recycling endosomes and efficiently recycles back to the plasma membrane in HeLaCIITA cells as well as in APCs (28). Although a direct link between pMHC-II recycling and ubiquitination has not been established, it is curious to note that overexpression of March-I promotes the re-distribution of MHC-II from early endocytic compartments to terminal lysosomes (16) and also that MHC-II co-localizes with recycling $\mathrm{TfR}^{+}$endosomes more in March-I-deficient B cells as compared to WT B cells (10). Furthermore, overexpression of the related MARCH family member MARCH-8 alters the itinerary of proteins internalized by clathrin-independent endocytosis from recycling endosomes to terminal lysosomes (29), leading to the possibility that ubiquitination of pMHC-II by March-I serves to 
limit recycling and promote lysosomal degradation of pMHC-II complexes.

\section{ACKNOWLEDGMENTS}

Our work cited in this mini-review was supported by the Intramural Research Program of the National Institutes of Health.

\section{REFERENCES}

1. Trombetta ES, Mellman I. Cell biology of antigen processing in vitro and in vivo. Annu Rev Immunol (2005) 23:975-1028. doi:10.1146/annurev.immunol. 22.012703.104538

2. Turley SJ, Inaba K, Garrett WS, Ebersold M, Unternaehrer J, Steinman RM, et al. Transport of peptide-MHC class II complexes in developing dendritic cells. Science (2000) 288:522-7. doi:10.1126/science.288.5465.522

3. Inaba K, Turley S, Iyoda T, Yamaide F, Shimoyama S, Reis e Sousa C, et al. The formation of immunogenic major histocompatibility complex class II-peptide ligands in lysosomal compartments of dendritic cells is regulated by inflammatory stimuli. J Exp Med (2000) 191:927-36. doi:10.1084/jem.191.6.927

4. Boes M, Cerny J, Massol R, Op den Brouw M, Kirchhausen T, Chen J, et al. T-cell engagement of dendritic cells rapidly rearranges MHC class II transport. Nature (2002) 418:983-8. doi:10.1038/nature01004

5. Chow A, Toomre D, Garrett W, Mellman I. Dendritic cell maturation triggers retrograde $\mathrm{MHC}$ class II transport from lysosomes to the plasma membrane. Nature (2002) 418:988-94. doi:10.1038/nature01006

6. Cella M, Engering A, Pinet V, Pieters J, Lanzavecchia A. Inflammatory stimuli induce accumulation of MHC class II complexes on dendritic cells. Nature (1997) 388:782-7. doi:10.1038/42030

7. West MA, Wallin RP, Matthews SP, Svensson HG, Zaru R, Ljunggren HG, et al. Enhanced dendritic cell antigen capture via toll-like receptor-induced actin remodeling. Science (2004) 305:1153-7. doi:10.1126/science.1099153

8. van Niel G, Wubbolts R, Ten Broeke T, Buschow SI, Ossendorp FA, Melief $\mathrm{CJ}$, et al. Dendritic cells regulate exposure of MHC class II at their plasma membrane by oligoubiquitination. Immunity (2006) 25:885-94. doi:10.1016/ j.immuni.2006.11.001

9. Shin JS, Ebersold M, Pypaert M, Delamarre L, Hartley A, Mellman I. Surface expression of MHC class II in dendritic cells is controlled by regulated ubiquitination. Nature (2006) 444:115-8. doi:10.1038/nature05261

10. Matsuki Y, Ohmura-Hoshino M, Goto E, Aoki M, Mito-Yoshida M, Uematsu $\mathrm{M}$, et al. Novel regulation of MHC class II function in B cells. EMBO J (2007) 26:846-54. doi:10.1038/sj.emboj.7601556

11. Bartee E, Mansouri M, Hovey Nerenberg BT, Gouveia K, Fruh K. Downregulation of major histocompatibility complex class I by human ubiquitin ligases related to viral immune evasion proteins. J Virol (2004) 78:1109-20. doi:10.1128/JVI.78.3.1109-1120.2004

12. Jabbour M, Campbell EM, Fares H, Lybarger L. Discrete domains of MARCH1 mediate its localization, functional interactions, and posttranscriptional control of expression. J Immunol (2009) 183:6500-12. doi:10.4049/jimmunol.0901521

13. Ishido S, Matsuki Y, Goto E, Kajikawa M, Ohmura-Hoshino M. MARCHI: a new regulator of dendritic cell function. Mol Cells (2010) 29:229-32. doi:10.1007/s10059-010-0051-x

14. Walseng E, Furuta K, Bosch B, Weih KA, Matsuki Y, Bakke O, et al. Ubiquitination regulates $\mathrm{MHC}$ class II-peptide complex retention and degradation in dendritic cells. Proc Natl Acad Sci U S A (2010) 107:20465-70. doi:10.1073/pnas.1010990107

15. Thibodeau J, Bourgeois-Daigneault MC, Huppe G, Tremblay J, Aumont A, Houde $\mathrm{M}$, et al. Interleukin-10-induced MARCH1 mediates intracellular sequestration of MHC class II in monocytes. Eur J Immunol (2008) 38:1225-30. doi:10.1002/eji.200737902

16. De Gassart A, Camosseto V, Thibodeau J, Ceppi M, Catalan N, Pierre P, et al MHC class II stabilization at the surface of human dendritic cells is the result of maturation-dependent MARCH I down-regulation. Proc Natl Acad Sci U S A (2008) 105:3491-6. doi:10.1073/pnas.0708874105
17. Wilson JE, Katkere B, Drake JR. Francisella tularensis induces ubiquitindependent major histocompatibility complex class II degradation in activated macrophages. Infect Immun (2009) 77:4953-65. doi:10.1128/IAI.00844-09

18. Bourgeois-Daigneault MC, Thibodeau J. Autoregulation of MARCH1 expression by dimerization and autoubiquitination. J Immunol (2012) 188:4959-70. doi:10.4049/jimmunol.1102708

19. Galbas T, Steimle V, Lapointe R, Ishido S, Thibodeau J. MARCH1 downregulation in IL-10-activated B cells increases MHC class II expression. Cytokine (2012) 59:27-30. doi:10.1016/j.cyto.2012.03.015

20. Tze LE, Horikawa K, Domaschenz H, Howard DR, Roots CM, Rigby RJ, et al. CD83 increases MHC II and CD86 on dendritic cells by opposing IL-10driven MARCH1-mediated ubiquitination and degradation. J Exp Med (2011) 208:149-65. doi:10.1084/jem.20092203

21. Walseng E, Furuta K, Goldszmid RS, Weih KA, Sher A, Roche PA. Dendritic cell activation prevents MHC class II ubiquitination and promotes MHC class II survival regardless of the activation stimulus. J Biol Chem (2010) 285:41749-54. doi:10.1074/jbc.M110.157586

22. Garrett WS, Chen LM, Kroschewski R, Ebersold M, Turley S, Trombetta S, et al Developmental control of endocytosis in dendritic cells by Cdc42. Cell (2000) 102:325-34. doi:10.1016/S0092-8674(00)00038-6

23. Sallusto F, Cella M, Danieli C, Lanzavecchia A. Dendritic cells use macropinocytosis and the mannose receptor to concentrate macromolecules in the major histocompatibility complex class II compartment: downregulation by cytokines and bacterial products. J Exp Med (1995) 182:389-400. doi:10.1084/jem.182.2. 389

24. Huang F, Goh LK, Sorkin A. EGF receptor ubiquitination is not necessary for its internalization. Proc Natl Acad Sci U S A (2007) 104:16904-9. doi:10.1073/pnas.0707416104

25. Haugsten EM, Malecki J, Bjorklund SM, Olsnes S, Wesche J. Ubiquitination of fibroblast growth factor receptor 1 is required for its intracellular sorting but not for its endocytosis. Mol Biol Cell (2008) 19:3390-403. doi:10.1091/mbc.E07-12-1219

26. Ma JK, Platt MY, Eastham-Anderson J, Shin JS, Mellman I. MHC class II distribution in dendritic cells and B cells is determined by ubiquitin chain length. Proc Natl Acad Sci U S A (2012) 109:8820-7. doi:10.1073/pnas.1202977109

27. Reid PA, Watts C. Cycling of cell-surface MHC glycoproteins through primaquine-sensitive intracellular compartments. Nature (1990) 346:655-7. doi:10.1038/346655a0

28. Walseng E, Bakke O, Roche PA. Major histocompatibility complex class IIpeptide complexes internalize using a clathrin- and dynamin-independent endocytosis pathway. J Biol Chem (2008) 283:14717-27. doi:10.1074/jbc M801070200

29. Eyster CA, Cole NB, Petersen S, Viswanathan K, Fruh K, Donaldson JG MARCH ubiquitin ligases alter the itinerary of clathrin-independent cargo from recycling to degradation. Mol Biol Cell (2011) 22:3218-30. doi:10.1091/mbc. E10-11-0874

Conflict of Interest Statement: The authors declare that the research was conducted in the absence of any commercial or financial relationships that could be construed as a potential conflict of interest.

Received: 30 July 2013; accepted: 28 October 2013; published online: 13 November 2013.

Citation: Cho K-J and Roche PA (2013) Regulation of MHC class II-peptide complex expression by ubiquitination. Front. Immunol. 4:369. doi: 10.3389/fimmu.2013.00369 This article was submitted to Antigen Presenting Cell Biology, a section of the journal Frontiers in Immunology.

Copyright (c) 2013 Cho and Roche. This is an open-access article distributed under the terms of the Creative Commons Attribution License (CC BY). The use, distribution or reproduction in other forums is permitted, provided the original author(s) or licensor are credited and that the original publication in this journal is cited, in accordance with accepted academic practice. No use, distribution or reproduction is permitted which does not comply with these terms. 